\title{
Design And Implementation Of Remote Digital Oscilloscopes Calibration System
}

\author{
Hongping $\mathrm{WANG}^{1, \mathrm{a}}$, Jun DAI ${ }^{1, \mathrm{~b}}$ \\ 1 Section 94, PLA 91550, Dalian, China \\ arosapple2003@sina.com, bhangzg602544@163.com
}

\begin{abstract}
.
This paper proposed an implementation scheme of networked oscilloscope remote control system. Based on LabView platform, combined with remote control technology and network data transmission, an oscilloscope remote control system could be established. This paper analyzed the remote control system hardware structure, measuring instruments accessing mode, software development technology, and basic modules of control system.
\end{abstract}

Keywords: LabView digital oscilloscope remote control.

\section{Introduction}

Remote virtual instrument can get dynamic data and send control signals to remote end via LAN or Internet, this makes monitoring remote end on local PC machine possible. Network measurement calibration will be the development direction of measurement technology. To establish a remote virtual instrument measurement and control system using network technology, the key point is to realize the traditional measurement functions using virtual instrument technology. Via Internet we can complete remote control and data transmission, and this makes information collection, transmission and processing integration. It is a valuable tool for measuring, monitoring and other fields of scientific research.

Remote metering guarantee is the development direction of military metrology, many foreign metering mechanism, such as American NIST, British NPL, German PTB, Japan's NMIJ have achieved remote calibration for some instruments and physical quantity based network ${ }^{[1-2]}$. At present, network and 
LAN technology develop very rapidly, many measuring instruments, testing equipment have been equipped with a special interface (such as GPIB, USB, RS 232), this provide hardware condition for remote measurement and test.

Applying the virtual instrument technology and network technology, we can develop remote control software to realize computer control, data acquisition and processing. Such software provides convenience for technical staff to test the equipment, and provide a reliable basis for failure analysis, and improve the work efficiency of measurement and calibration.

\section{Establishing of measurement scheme}

This paper proposes a scheme of remote control system based on Web, taking the digital oscilloscope for example. The remote digital oscilloscope system consists of three parts: control server (field acquisition terminal), Web server, client (remote control terminal).The field acquisition terminal mainly completes the data acquisition, data distribution, and data saving to server database. Web server acts as a website, the client can access the website, get real-time and historical data. At the same time, the client uses virtual instrument application or browser to monitor network communication, receive and control server job data, process and analyze data, and do some other works, such as data storage and display, report generation and waveform print.

According to the status of remote measurement data flow and different test demand, we can apply C/S or B/S to establish a remote test system. C/S model suits for large data transmission flow, and has the advantages of high efficiency, reliable data integrity, and complete compatibility. As for small quantity data transmission, we can adopt B/S model. The remote test system functions include data communication module, user interaction module and remote instrument control module, data acquisition and network transmission module, analysis module and report generation module etc.

\section{The composition and technologies of the system}


Remote Virtual Instrument Technology. At present, the trend of measurement and control involves systematization and information integration, publishing and sharing measurement data in network is one of the key technologies of network based remote measurement.

Traditional measurement and calibration system operation is in the field. Both in situ calibration and offline calibration need disassembly and transportation, which brings inconvenience to military security. Via LAN or Internet we can realize remote measurement and control, effective and reasonable configuration of various resources. Combined with powerful network communication of virtual instrument and LabView, we can easily realize the remote measurement and control. In the development of LabView, it fully took into account the measurement and control system's network requirements, and provides rich network components. It not only includes the traditional network communication technology, NI also proposed Data socket technology designed for real-time transmission of measurement data.

There are several methods to achieve remote control in LabView:

1. Windows desktop's Remote connection function.

2. Data sharing using Data socket technology.

Data socket is a network measurement and control system development technology provided by NI Corporation, it can be used for data exchange, sharing and publishing real-time data, real-time data transmission between multiple applications within a computer or a network. Because of the Data socket is protocol, language and operating system independent, so it can be used in a variety of development environment, such as LabView, VB and VC. The Data Socket has the virtue of high security and reliability of data transmission. Data Socket can set client connection number, create user groups and users, and set user permissions. In addition, Data socket uses Web URL to illustrate the communication protocol and locate the data source. The user can select the type of communication protocol based on published data and network configuration. DSTP is the inner communication protocol, and any type of data can be transmitted based on it. 
According to the transmission protocol settings, data publishing program can be flexibly operated in computer, which suitable for real-time data transmission.

3. Network protocols

The TCP/IP protocol is basic Internet protocol, and is divided into the network interface layer, network layer, transport layer and application layer. Two different sub protocol templates have existed in LabView, you can create a data transmission connection between server and client.

4. Remote Panel Technology

Labview7.1 and above version contains Remote Panel, it allows users to realize remote control by directly operating server side's VI front panel on local machine, even by using a web browser.

5. Publishing on Web

LabView has the ability of publishing data on remote panel. We can operate remote VI on local machine, even via web page.

Function design. According to "GJB7691-2012 digital oscilloscope test procedures" and calibration process, we design measure system functions. There are four parts, including user management, device management, remote control, data processing. ${ }^{[3]}$

1. User management

This function includes user information management, authentication, and rights management. System administrators log off users, add new users and manage user information, authority. Users can update their own information and passwords.

\section{Device Management}

Users can manage device information and do some other operations.

\section{Remote control}

This function can be realized on a remote control device. We can complete set of virtual instrument function, simulate devices panel, and monitor real-time status of the test.

4. Data processing 
This function includes detection point setting, data judgment, and certificate generation. Data storage is also supported in this module. According to actual needs, we can choice the appropriate verification methods to set verification projects.

The structure of network and software development. Site measurement and digital oscilloscope should be equipped with intelligent interface to realize remote data transmission and computer control, such as GPIB, USB, PCI or VXI RS-232. Network remote calibration hardware system is shown as Fig.1. The system mainly includes the digital oscilloscope, measuring equipment, computer, network system and remote control PC.

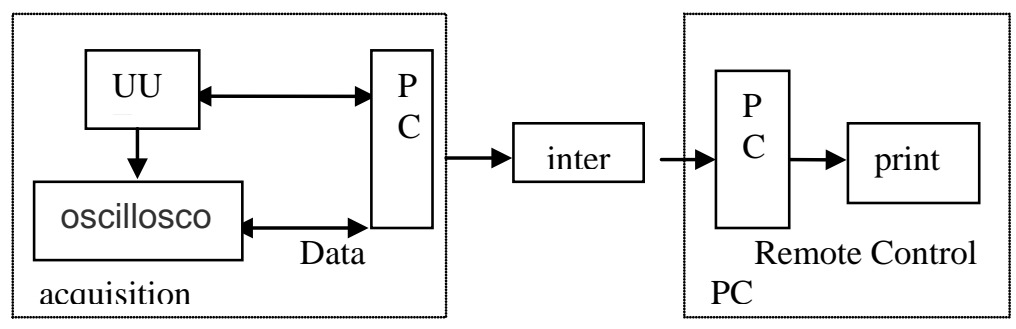

Fig.1 the system constitution

The digital oscilloscope's main function is to show signal characteristics in time domain of signal generator, power supply and so on, including signal frequency, signal cycle, the magnitude of the maximum and minimum values, peak to peak, RMS and signal DC component etc. We could use Visual C++, Visual Basic, a graphical programming language such as LabView, HPVEE to realize these functions. The measurement system process is shown in Fig.2. 


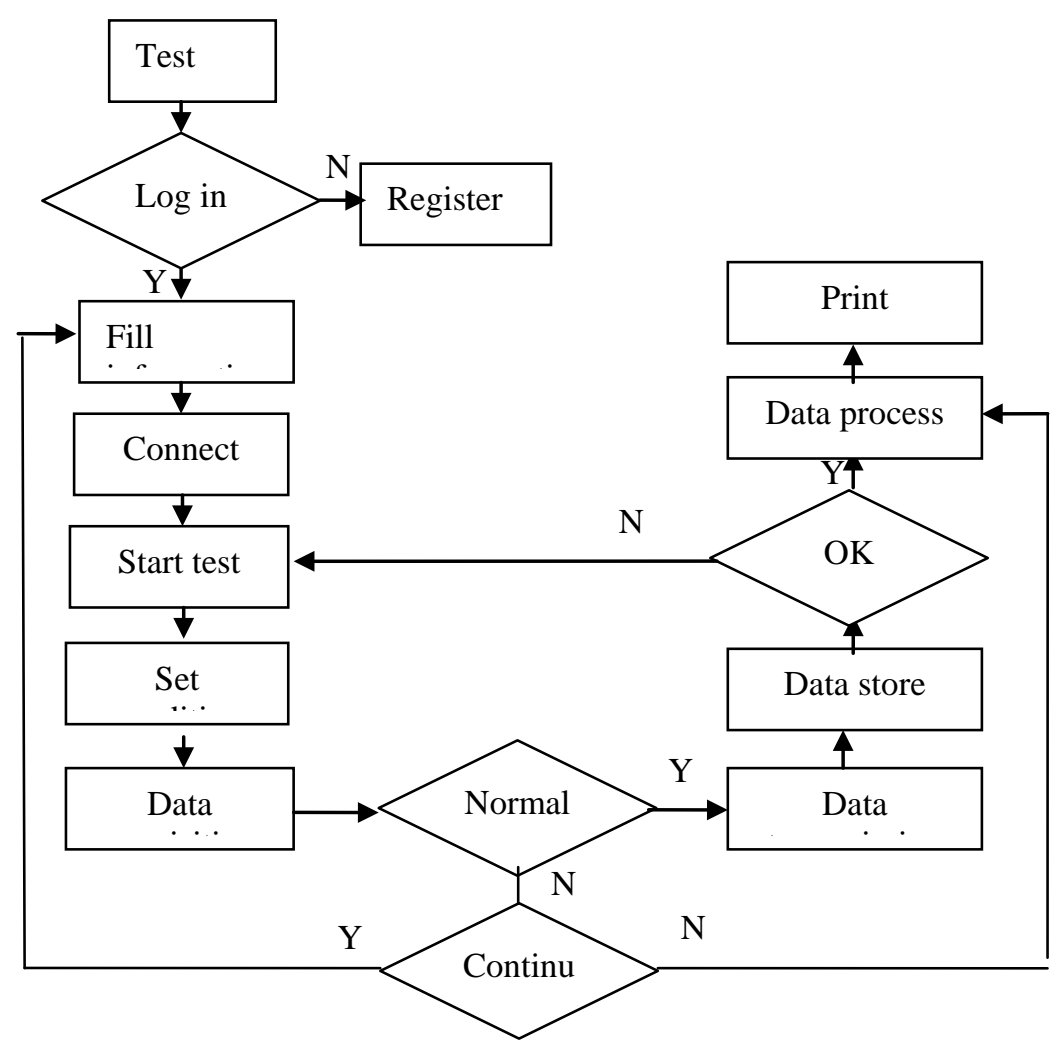

Fig.2 system process diagram

User can login in test Web page by checking username and password, new user must register first. According to test condition, fill the instrument information and test project. After connecting the test circuit, submitted collection data to the remote control laboratory. We can judge the correctness of data collection, if meeting the requirements, and we calculate the data and generate report.

Digital oscilloscope function should be set according to the common oscilloscope function: double channel waveform input, trigger mode, sampling methods, coupled mode choice of operation control. Measurement functions include: voltage amplitude, peak to peak value, the signal frequency, cycle, decrease time, rise time, correlation analysis and the basic function of waveform parameters measurement. ${ }^{[4]}$

Test data must be pretreated before performing data processing. This can purify signals and improve the accuracy of measurement. ${ }^{[5]}$ 
The user information, the measured equipment information and remote data information is stored in the server as data file. User can use LabSQL or LabView Database Connectivity Tool Set to realize the effective management and use of data.

\section{Conclusions}

Remote control system is introduced in this paper, through network we can build a stable, reliable test platform to solve the problem of disassembly, transportation, reduce the work intensity of engineering testing personnel, and improve the test efficiency. The modular structure design of remote measurement system can be transplanted for other similar remote measurement, calibration. This paper provides a reference for the in situ calibration for weapon equipment.

\section{References}

[1] ANDERSON W, OLEHAM N, PARKER M. An Internet-based video conferencing system supporting metrology. Proceedings of the NCSL Workshop\&Symposium, Washington. DC, 2000.

[2] JURCEVIC M, BORSIC M, MALARIC R. Internet-enabled calibration services: design of a secure calibration system. IEEE Transactions on Instrumentation and Measurement, 2008, 57(9):2012-2018.

[3] Xieqi, Guyimin. Dynamic electrical parameters measurement and analysis system based on LabView. Instrument Technique and Sensor, [J] 2009(7):54-56.

[4] Weibo Li. Network virtualization technology integrated system of electronic measuring instruments. [D] Jilin: Jilin University 2012.

[5] Aihu Cao. Control system based on virtual instrument development and research. [D] Shenyang: Northeastern University 2010. 